\title{
SURFACE SELF-DIFFUSION OF IRIDIUM: FIELD ELECTRON EMISSION STUDY
}

\author{
G. Antczak, M. Blaszczyszyn and R. Beaszczyszyn \\ Institute of Experimental Physics, University of Wrocław \\ Pl. M. Borna 9, 50-204 Wrocław, Poland
}

(Received May 18, 2000; revised version July 17, 2000)

\begin{abstract}
The self-diffusion of iridium was studied by means of field electron microscopy. The measurements, based on the well-known process of surface build-up, were carried out under the UHV conditions within the temperature range of $790-935 \mathrm{~K}$. The activation energy for the diffusion was determined to be equal to $2.10 \pm 0.10 \mathrm{eV} /$ atom $(48.4 \pm 2.3 \mathrm{kcal} / \mathrm{mol})$. This value is compared with activation energies for self-diffusion on other metal surfaces, as well as with those for self-diffusion of single iridium atoms and clusters on iridium, known from the field ion microscopy measurements.
\end{abstract}

PACS numbers: 61.16.-d, 68.35.Fx, 68.55.-a, 68.90.+g

\section{Introduction}

Surface diffusion of metals is an important topic from both technological and scientific points of view. There are several standard methods to study this process, depending on the nature of investigated systems and physical or chemical problems $[1,2]$ to be solved. One of them is the field electron microscopy (FEM) of multi-faced surface of the field emitter tip. To study the self-diffusion by means of FEM, the build-up procedure was applied as described by Sokolskaya [3] and Bettler and Charbonnier [4]. They demonstrated that annealing of a tungsten emitter tip at a high temperature in the presence of a high electric field resulted in a geometric deformation of the emitter tip. As a final result a nearly polyhedral shape bounded by certain large planar facets with low Miller indices was obtained. During the heating of the built-up tip at the field taken off, the transition to the equilibrium shape of the tip crystal occurred, the field emission images accompanying the transition could be observed, and the high voltage changes at a constant total emission current detected.

Little FEM work on iridium self-diffusion has been reported. The first FEM investigation of iridium self-diffusion was reported by Brenner in 1964 during the 11th Field Emission Symposium [5]. Recently FEM has been applied to study the faceting and steady-state shape of field emitter tips for such adsorbate-adsorbent 
systems as: Rh/W, Pd/W [6], Pt/W, Pd/Mo and Pt/Ir [7]. The extensive faceting of planar $\mathrm{W}(111)$ and $\mathrm{Mo}(111)$ surfaces induced by metallic adsorbates such as $\mathrm{Rh}, \mathrm{Pd}, \mathrm{Ir}, \mathrm{Pt}$ has been carefully studied by Madey and co-workers [8].

The present study of the self-diffusion of iridium has been undertaken to get a better understanding of the faceting process. The effect of metal adsorbate on the substrate-metal self-diffusion is now under investigation, and the results will be reported in the forthcoming paper.

\section{Experimental}

\subsection{Apparatus}

The experiment was performed in the field emission (FE) glass tubes attached to a stainless-steel vacuum system. The tubes were equipped with a BayardAlpert ionization gauge and liquid nitrogen cooled $\mathrm{Ti}$ getter. The vacuum system provided a total base pressure below $1 \times 10^{-10}$ Torr during measurements. The $\mathrm{Ir}$ emitters were made of wire of $0.15 \mathrm{~mm}$ in diameter (Goodfellow), purity $99.9 \%$. The emitter was spot welded onto a tungsten wire loop. The Ir wire was pre-annealed at $800-900 \mathrm{~K}$ in oxygen, at $\sim 7.5 \times 10^{-9}$ Torr, for 1 hour (as suggested in Ref. [9]), and then outgassed under UHV conditions. The temperature of outgasing was high enough for a distinct evaporation of iridium. Emitters were prepared using Melmed's procedures [10]. The Ir emitter tip was cleaned by the resistive heating of the emitter loop assembly. The temperature of the emitter tip was determined from the measurement of the resistance of the apex segment of the tungsten wire loop [11]. The FE images were recorded while the imaging voltage at a fixed emission current was measured. The FE patterns could be observed directly on the screen of the microscope or by means of a TV camera and video monitor. The patterns were video-tape recorded and then computer analysed.

\subsection{Experimental procedure}

. In order to determine the activation energy $E$ for the self-diffusion of Ir the build-up method was employed $[3,4,12]$ in the following stages:

(i) Thermal cleaning of Ir tip

The pattern in Fig. 1a represents a FE image, corresponding to the surface of a clean Ir emitter tip of a curvature radius of about $300 \mathrm{~nm}$. Such patterns were observed after annealing the emitter at various temperatures - in particular after intensive heating and flashing the sample to a temperature close to its melting point, and then rapid quenching to room temperature. The enhanced electron emission around the Ir $\{100\}$ planes appearing always in our FE patterns can be, in principle, attributed to the contamination of a sample following the suggestion given by Vanselow and co-workers (e.g. [13]) and Melmed [12] for Pt emitter tips. According to Melmed, the attaining of a clean surface of iridium tip is a difficult task [14]. Emission rings around the $\{100\}$ planes were also observed by Hashim and Jones [9]. We are not sure however, whether in our case this phenomenon is really caused by the contamination of the tip surface. The enhanced emission area 
in Fig. 1a has a square shape rather than a ring-like one. Such a square form of the $\operatorname{Ir}(100)$ surface top layer, with four sides parallel to the $\langle 100\rangle$ directions, was found for thermal equilibrium conditions in the field ion microscopy (FIM) studies (on the atomic scale) by Tsong and co-workers [15]. They showed that around the temperature of $400 \mathrm{~K}$, the lattice step atoms started to move along the $\langle 110\rangle$ steps of the crystal surface. Perhaps this effect (on a massive scale) is responsible for the enhanced local emission seen in Fig. 1a. This supposition should however be examined in additional experiments. Even if the sample is not completely clean, one can expect that the contamination is located only around the $\{100\}$ planes, and the remaining part of the surface is free of it. The annealing of the emitter tip at various temperatures did not change the pattern in comparison to that shown in Fig. 1a.

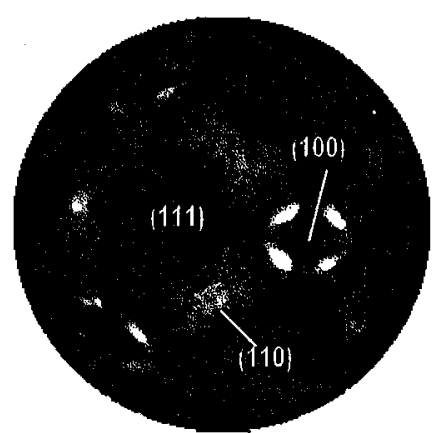

a) initial state

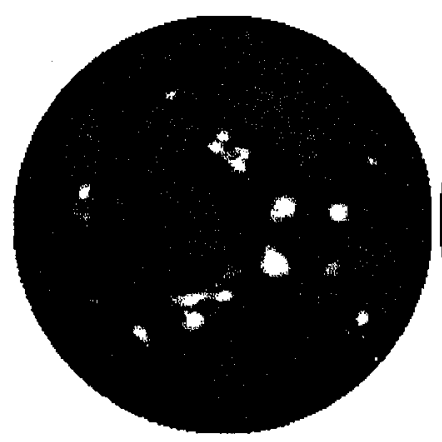

d) $t=110 \mathrm{~s}$

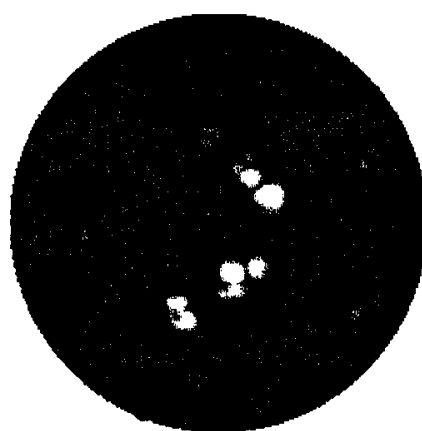

b) after "build-up"

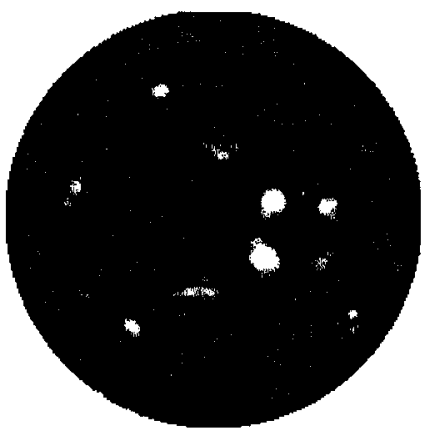

e) $\mathrm{t}=300 \mathrm{~s}$

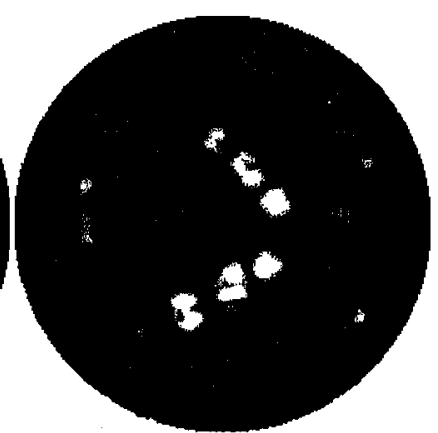

c) $t=20 \mathrm{~s}$

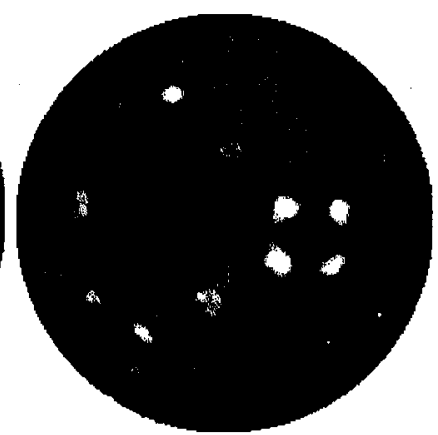

f) $t=5700 s$

Fig. 1. Typical sequence of the emission patterns of iridium: (a) before "build-up", (b) after "build-up", (c-f) during the self-diffusion at $T=865 \mathrm{~K}(t-$ diffusion time).

(ii) A geometric deformation of the tip, i.e. build-up, by heating for $30 \mathrm{~s}$ at $1023 \mathrm{~K}$ in the presence of a high electric field (FEM mode of operation, field strength $39 \mathrm{MV} / \mathrm{cm}$ )

Under these conditions the initially hemispherical tip surface was deformed into a polyhedral form shown in Fig. 1b. 
(iii) Measurements of imaging voltage $U$ at a fixed emission current as a function of the annealing time $t$ of the tip at constant temperatures $T$

The annealing was made in the absence of a high electric field, so the transition from a polyhedral form to a hemispherical tip surface could occur only as a result of the diffusion process. Figures $1 c-f$ present subsequent stages of the diffusion. The rate of the diffusion at various temperatures was determined on the basis of $U$ vs. $t$ dependences which are presented in Fig. 2.

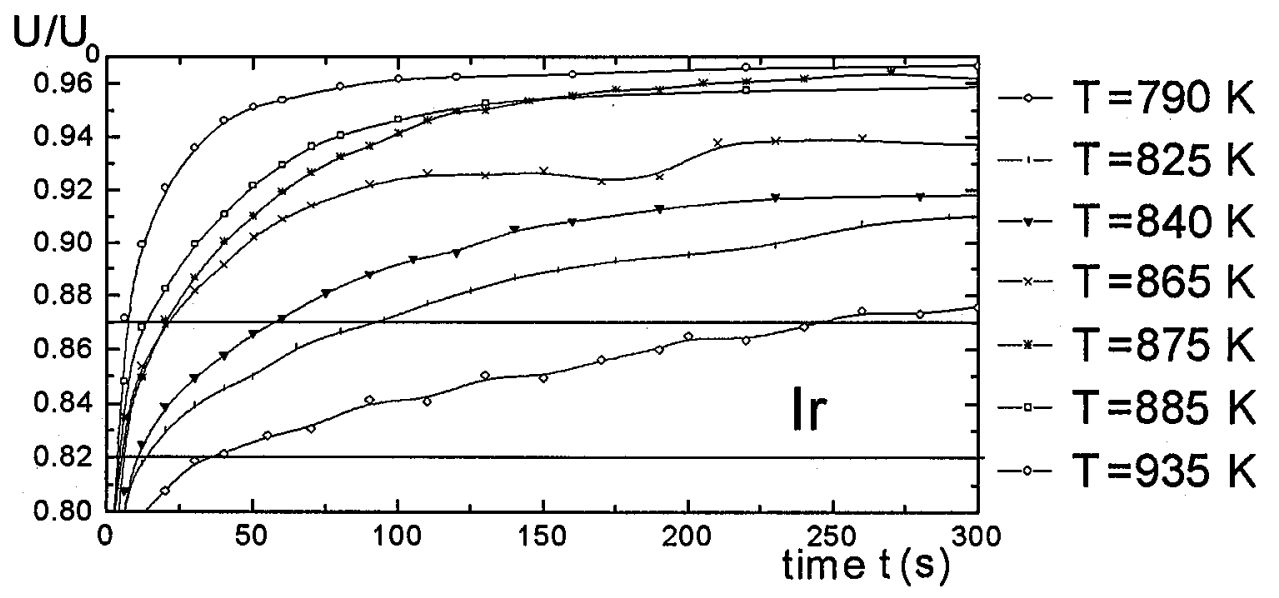

Fig. 2. Dependence of the normalized high voltage $U / U_{0}$ (for a constant total emission current, $I_{\mathrm{e}}=0.5 \mu \mathrm{A}$ ) versus self-diffusion time $t, T$ is the parameter of the curves, $U_{0}$ denotes the voltage before "build-up".

(iv) Determination of the activation energy for the surface self-diffusion from the Arrhenius-type plot

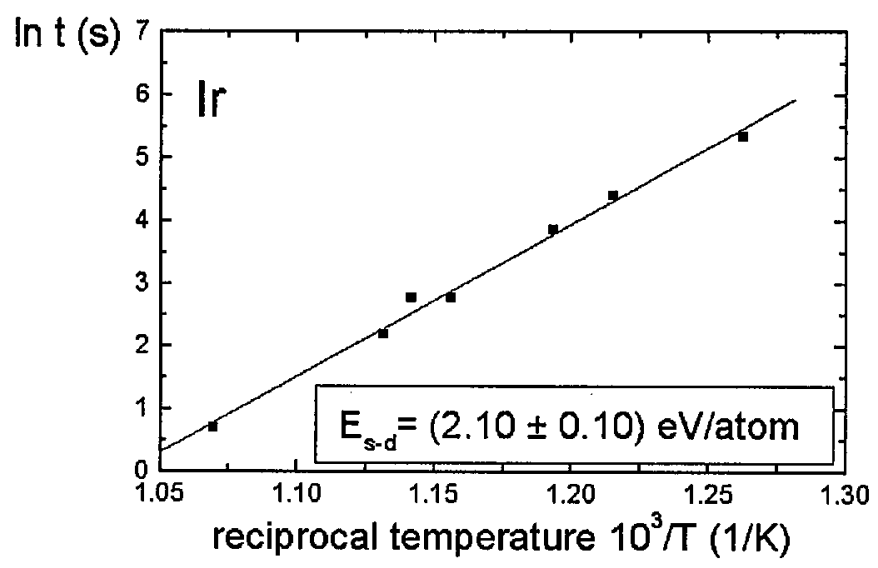

Fig. 3. Arrhenius plot for self-diffusion of iridium. 
The Arrhenius plot for the diffusion between the stages represented in Fig. 2 by horizontal lines, is shown in Fig. 3.

\section{Result and discussion}

The self-diffusion of iridium on a tip surface, investigated within the temperature range of 790-935 $\mathrm{K}$, proceeded with the average activation energy $E=2.10 \mathrm{eV} /$ atom $(48.4 \mathrm{kcal} / \mathrm{mol})$. While comparing patterns of Fig. $1 \mathrm{a}$ and b, it can be seen that during the process of built-up, the iridium atoms are transported mainly from the $\{111\}$ planes to the zones between the $\{100\}$ planes: the $\{111\}$ planes are growing and their edges become more prominent. During the heating of the emitter tip in the absence of a field, a restoration of the equilibrium shape of the tip occurs. The activation energy for diffusion determined in this work can be attributed to the diffusion in the region of the steps of $\{111\}$ faces.

Brenner in his FEM studies determined the activation energy for the self-diffusion of iridium, $E=2.39 \mathrm{eV} /$ atom $(55 \mathrm{kcal} / \mathrm{mol}$ ) [5] in the temperature range of $425-1400 \mathrm{~K}$. By means of FIM he also observed an appreciable diffusion of Ir atoms on the field evaporated surfaces over a distance not larger than a few angstroms (in the high-index facet regions) at temperatures above $450 \mathrm{~K}$ [16]. A continued heating leads to Ir atoms migration away from the edges of low-index planes causing a flattening of those regions. The activation energy determined in [16] for growth of the $\operatorname{Ir}\{111\}$ facet at $465-725 \mathrm{~K}$ was found to be $E=1.95 \mathrm{eV} /$ atom $(45 \mathrm{kcal} / \mathrm{mol})$ [16]. Comparison with the results for other (fcc) metals is presented in the Table.

TABLE

Summary of the results.

\begin{tabular}{l|c|c|c|c|c}
\hline \hline & $\mathrm{Ni}^{a}$ & $\mathrm{Ni}^{b}$ & $\mathrm{Pt}^{a}$ & $\mathrm{Ir}^{c}$ & $\mathrm{Ir}^{d}$ \\
\hline$E[\mathrm{kcal} / \mathrm{mol}]$ & $21.4 \pm 2.1$ & $24.9 \pm 2.6$ & $29.5 \pm 3.0$ & $55 \pm 5$ & $48.4 \pm 2.3$ \\
Temp. range [K] & $510-752$ & $562-612$ & $552-848$ & $425-1400$ & $792-935$ \\
\hline
\end{tabular}

${ }^{a}$ A.J. Melmed [12]; ${ }^{b}$ M. Błaszczyszyn [21]; ${ }^{c}$ S. S. Brenner [5]; ${ }^{d}$ this paper.

FIM studies provided also interesting results on the self-diffusion of single atoms and small clusters of Ir on the low-index planes of the Ir microcrystal: $\{111\}$, $\{100\}$ and $\{110\}$. Two different diffusion mechanisms have been found: diffusion by the jumping of surface atoms or by their exchange [17-20]. The activation energy and mechanism depend on the substrate structure and size of the cluster. In general, the activation energy for diffusion under such conditions is lower than $1 \mathrm{eV} /$ atom. Our result indicates that the transport of the material in "extensive self-diffusion" observed in FEM, requires a much higher activation energy than that during the diffusion of single atoms or small clusters on an atomically perfect substrate [18]. 


\section{Conclusion}

It is shown that the multi-atomic self-diffusion of iridium proceeds with an average activation energy $E=2.10 \pm 0.10 \mathrm{eV} /$ atom $(48.4 \pm 2.3 \mathrm{kcal} / \mathrm{mol})$. A time required for the displacement of the surface atoms on a distance of $\sim 50 \AA$ is estimated to be of the order of seconds at $940 \mathrm{~K}$ whereas at $790 \mathrm{~K}$ it amounts to a few minutes.

\section{Acknowledgment}

G.A. acknowledges support by the Wrocław University, grant No. 2016/W/IFD/99.

\section{References}

[1] R. Gomer, Rep. Prog. Phys. 53, 917 (1990).

[2] A.G. Naumovets, Yu.S. Vedula, Surf. Sci. Rep. 200, 365 (1985).

[3] I.L. Sokolskaya, Soviet Phys. (Tech. Phys.) 1, 1147 (1956).

[4] P.C. Bettler, F.M. Charbonnier, Phys. Rev. 119, 85 (1960).

[5] S.S. Brenner, Abstracts of 11th Field Emission Symposium, Cambridge (England) 1964.

[6] K. Pelhos, T.E. Madey, R. Błaszczyszyn, Surf. Sci. 426, 61 (1999).

[7] G. Antczak, T.E. Madey, M. Błaszczyszyn, R. Błaszczyszyn, in preparation.

[8] T.E. Madey, C.-H. Nien, K. Pelhos, J.J. Kołodziej, I.M. Abdelrehim, H.-S. Tao, Surf. Sci. 438, 191 (1999).

[9] K.I. Hashim, J.P. Jones, Thin Solid Films 245, 89 (1994).

[10] A.J. Nam, A. Teren, T.A. Lusby, A.J. Melmed, J. Vac. Sci. Technol. B 13, 1556 (1995); A.J. Melmed, J. Vac. Sci. Technol. B 9, 601 (1991).

[11] C. Workowski, J. Phys. E. 10, 538 (1977).

[12] A.J. Melmed, J. Appl. Phys. 38, 1885 (1967).

[13] M. Mundschau, R. Vanselov, Surf. Sci. 155, 121 (1985).

[14] A.J. Melmed, private information.

[15] Ch. Chen, T.T. Tsong, Surf. Sci. 336, L735 (1995).

[16] S.S. Brenner, Surf. Sci. 2, 496 (1964).

[17] Ch. Chen, T.T. Tsong, Phys. Rev. Lett. 64, 3147 (1990).

[18] G.I. Kellog, Surf. Sci. Rep. 21, 1 (1994).

[19] A. Friedl, O. Schutz, K. Müller, Surf. Sci. 266, 24 (1992).

[20] T-Y. Fu, T.T. Tsong, Surf. Sci. 421, 157 (1999).

[21] M. Błaszczyszyn, Surf. Sci. 136, 103 (1983). 